\title{
Deep brain stimulation of the orbitofrontal cortex reduces perseverative behavior induced by quinpirole in rats
}

\author{
Abraham Flores-Vargas ${ }^{1 *}$, Cuauhtémoc Campos-García-Rojas ${ }^{2}$ and Fiacro Jiménez-Ponce ${ }^{3}$ \\ ${ }^{1}$ Department of Anesthesiology, General Hospital of México "Dr. Eduardo Liceaga"; ${ }^{2}$ Department of Experimental Surgery, General Hospital of México \\ "Dr. Eduardo Liceaga"; ${ }^{3}$ Reserch Department, Instituto Nacional de Seguridad y Servicios Sociales de los Trabajadores del Estado, Mexico City, \\ Mexico
}

\begin{abstract}
Introduction: Different rodent models of obsessive-compulsive disorder (OCD) have been used with the purpose for understanding this mental disease; in a previous article, we described quinpirole (QP) as a dopaminergic inductor of perseverative behavior. Objective: The objective of this study was to evaluate the decrease of QP-induced perseverative behavior observed in an open field after unilateral deep brain stimulation-low frequency (DBS-LF) into the orbitofrontal cortex (OFC) in Wistar rats. Materials and Methods: $A$ total of 40 adult male Wistar rats were divided into three groups, each group underwent pharmacological treatment: sodium chloride ( $\mathrm{NaCl})$, QP, and clomipramine (CMP), these groups were not implanted with an electrode. An additional group was implanted into OFC Sham (SH), and after behavior evaluation, unilateral DBS-LF (6 Hz) was applied during night time for 5 min each side; first left side and after right side. Results: Distance traveled significantly decreased in the 5 min observed between $Q P$ and $\mathrm{NaCl}(p=0.016)$, and a significant difference was found between $\mathrm{NaCl}$ and $\mathrm{SH}(p=0.020)$. There was no significant difference between $\mathrm{NaCl}$ and $\mathrm{CMP}(p=0.582)$ or $\mathrm{NaCl}$ and DBS-LF ( $p=0.829)$. No significant difference between DBS left side and right side was found ( $p=1.00)$. Conclusions: Unilateral DBS of the OFC prevents perseverative behavior in an open field, induced by $Q P$.
\end{abstract}

Key words: Deep brain stimulation. Quinpirole. Orbitofrontal cortex. Perseverative behavior.

\section{Introduction}

The obsessive-compulsive disorder (OCD) rodent model can be pharmacologically induced with the following drugs, i.e., RU24969, 8-OH-DPAT, neonatal clomipramine (CMP), and quinpirole $(\mathrm{QP})^{1}$. The $\mathrm{OCD}$ is compared to perseverative behavior in rats and is evaluated through an open field and a T-maze test. QP is a D2/D3 dopamine receptor agonist ${ }^{1}$, and when chronically applied in rats, it can induce perseverative behavior. This effect is a model of OCD2. Dopamine induces motor functions in different regions of the central nervous system, involving different brain circuits. The rats motor activity decreases through the activation of the D2 receptors in striatopallidal neurons ${ }^{3}$. Repeated administration of dopaminergic D2 agonists produces receptor hypersensitivity, which conditions a receptor state known as high-affinity (D2High). This mechanism is responsible for some behaviors generated after administering $\mathrm{QP}^{4}$. Receptor hypersensitization is known to occur in some psychiatric disorders such as schizophrenia, OCD, and motor dyskinesias ${ }^{4}$. Similar behaviors are observed in animals sensitized with QP, such as stereotypies, antinociception, locomotor 
activity, alterations in memory, and learning. QP can decrease locomotor activity in the first 15 min after it is administered and it increases motor activity after the $2^{\text {nd }} \mathrm{h}$ of application ${ }^{3}$.

Dvorkin, in 2010, suggested that QP may drive the vigor of the checking behavior by inhibition of nucleus accumbens neurons, and it can be a site for the negative feedback control of checking5; nevertheless, the mechanistic understanding of cellular and molecular changes occurring in the orbitofrontal cortex (OFC) is unclear $^{6}$. Recent studies have been published using optogenetics to examine the contribution of OFC inputs to the striatum, in the evolution of perseverative grooming ${ }^{7,8}$; however, the pathologic changes that may alter these inputs in OCD are unknown ${ }^{6}$.

de Haas et al. observed an increase of dopamine into the nucleus accumbens in perseverative rats treated with $\mathrm{QP}^{9}$. Schmidt and colleagues found that chronic treatment with QP produces perseverative behavior and increment of locomotion in rats; these findings were not observed in the control group where D-amphetamine was administered ${ }^{10}$.

In 2007, McCracken and Grace applied deep brain stimulation (DBS) high frequency $(130 \mathrm{~Hz})$ into the OFC decreasing OCD behavior by reducing the neuronal activity and inhibiting the activation of the antidromic conduction of collateral axons in the corticostriatal region ${ }^{11}$. These findings suggest that using high frequency in the subthalamic nucleus can be used as a therapeutic strategy for $\mathrm{OCD}^{12}$. In addition, high-frequency DBS of the entopeduncular nucleus and globus pallidus decreases perseverative behavior in rats ${ }^{13}$. Previously, we have published information about lesion or low-frequency DBS into reticular nucleus of thalamus and how it decreases perseverative behavior in rats induced by $8 \mathrm{OH}-\mathrm{DPAT}^{14}$.

On the other hand, different anatomical structures such as the anterior limb of the internal capsule, nucleus accumbens, subthalamic nucleus, ventral striatum, and the inferior thalamic peduncle have been involved in the treatment of OCD patients ${ }^{15,16}$. Fitzgerald and Segrave suggest that DBS in the nucleus accumbens or ventral striatum could modulate the amygdala - basal ganglia - prefrontal circuitry that is abnormally active in mood and anxiety disorders ${ }^{17}$. Sturm, in 2003, reported that unilateral high-frequency DBS into accumbens nucleus could decrease OCD symptoms ${ }^{18}$ that information differs to the rest of the authors who had applied bilateral DBS. Our team had published the decreasing effect of bilateral DBS of inferior thalamic peduncle in OCD symptoms of eight patients ${ }^{15,19}$.
The aim of this study is to evaluate the effect of DBS low-frequency (DBS-LF) bilateral on OFC in Wistar rats with perseverative behavior induced by QP through an open field.

\section{Materials and methods}

\section{Subjects}

To perform this study, healthy male Wistar rats that weight between 250 and $350 \mathrm{~g}$ were selected, none of which showed abnormal motor behavior by rotarod test. Rats had free access to food and water, they were single housed and kept in a 12:12 light-dark cycle at a temperature-controlled room; to avoid bias caused by the circadian cycle of rats, DBS was performed at night ${ }^{20}$. The entire management of animals was done according to the Mexican Official Norm NOM-062-ZOO-1999. This experiment was approved by the Research and Ethics Committee of the General Hospital of México "Dr. Eduardo Liceaga".

48 rats were included in the experiment; however, eight rats met the exclusion criteria: infection at the surgical site after electrode implantation (four rats), death during the experimental maneuver (two rats), and rats which ripped off the electrode (two rats).

\section{Drugs}

All groups of rats were induced to perseverative behavior by administering QP hydrochloride (Sigma-Aldrich by Eli Lilly and Company, Indianapolis, USA) chronically ( $0.5 \mathrm{mg} / \mathrm{kg}$ twice weekly for 5 weeks) subcutaneously at the cross-skull region, with the exception, of the sodium chloride $(\mathrm{NaCl})$ group, which was injected with the same amount of the volume of $0.9 \% \mathrm{NaCl}$ solution. For the surgical implantation of the electrode, the animals were placed in a stereotactic apparatus Kopf instruments, model 960 (David Kopf Instruments Tujunga Ca. USA).

Surgery was performed under an anesthetic procedure using Ketamine (Anesket, PISA Mexico) $0.1 \mathrm{mg} / \mathrm{kg}$ administered intramuscularly and Acepromazine (Calmivet, Vétoquinol, France) $0.5 \mathrm{mg} / \mathrm{kg}$ administered intramuscularly; no analgesic or antibiotic was administered to avoid bias caused by drug interaction.

\section{Apparatus}

Perseverative behavior was correlated with motor displacement (distance traveled in centimeters [cm]) by the rat, the further distance traveled the least perseverative 


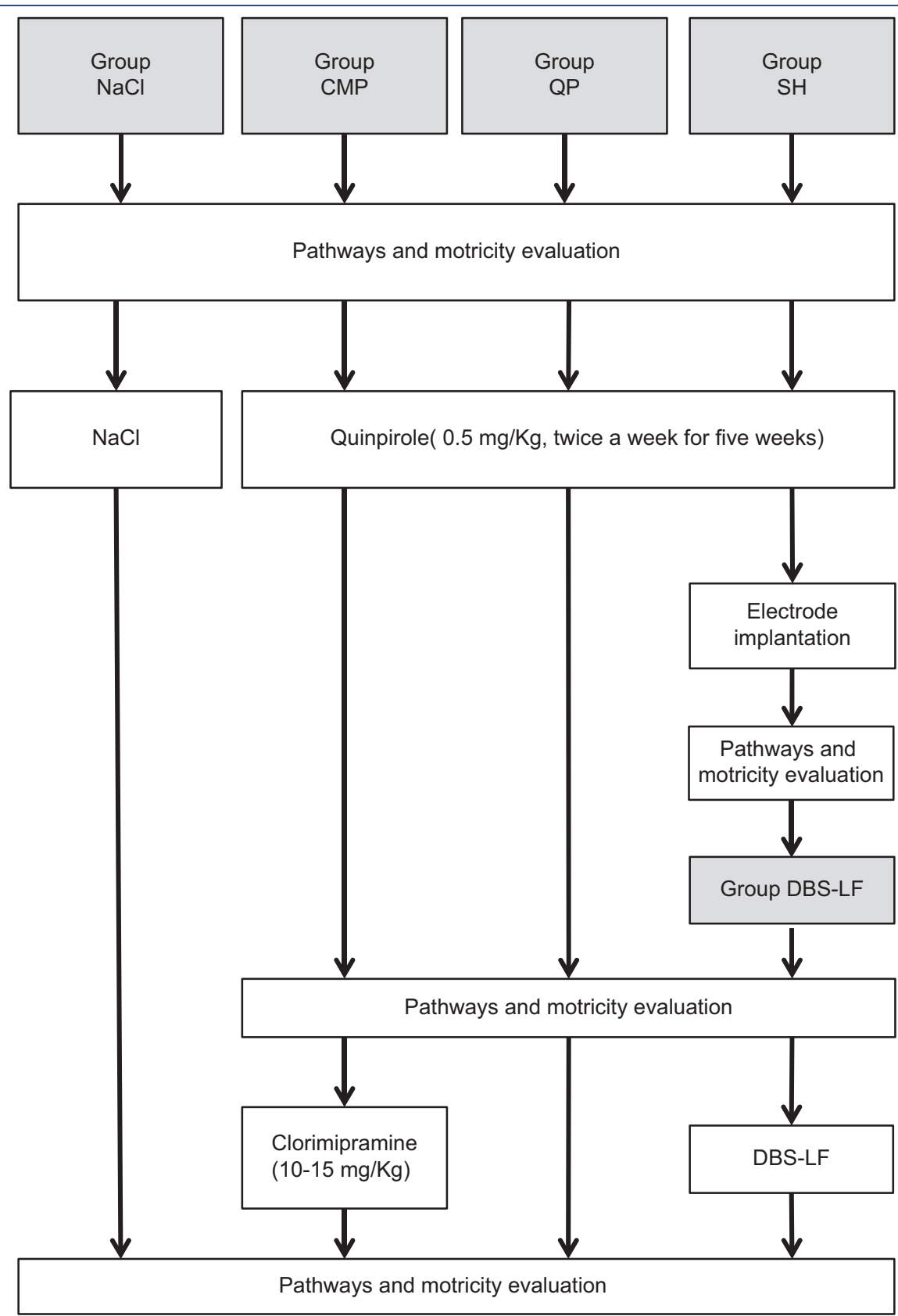

Figure 1. Treatment and procedures applied to 40 animals, each group with $n=12$.

the rat was, the distance traveled in $5 \mathrm{~min}$ in $\mathrm{cm}$ was used as the main output variable. The apparatus used to evaluate this behavior was an open field plastic box with the following dimensions: $160 \mathrm{~cm}$ long $\times 160 \mathrm{~cm}$ wide and $60 \mathrm{~cm}$ high, subdivide into 25 regular quadrants.

\section{Experimental design}

Four groups were formed, the group treated with $\mathrm{NaCl}$ was named $\mathrm{NaCl}$, the group treated with QP was named QP, and the group previously sensitized with QP and subsequently treated with CMP was named CMP. Finally, the group Sham (SH) (implanted with electrodes) in OFC without DBS was assessed in an open field. After $24 \mathrm{~h}$, the same group was submitted to unilateral DBS-LF into the OFC (10.5 V, 450 micro sec, $761 \mathrm{~W}$, and $6 \mathrm{~Hz}$ ) first LF-left side and then LFright side for 5 min each session, DBS was performed at night (Fig. 1). A stereotactic apparatus (Kopf instruments, model 960) was used for implantation. 
Table 1. Differences $(\Delta)$ between $\mathrm{NaCl}$ versus other groups

\begin{tabular}{|c|c|c|c|}
\hline & $\Delta$ & & \multirow[t]{2}{*}{$p$} \\
\hline & \multicolumn{2}{|l|}{$\mathrm{NaCl}$} & \\
\hline \multirow[t]{5}{*}{ Distance traveled in $\mathrm{cm} / 5 \mathrm{~min}$} & QP & -390.0 & 0.016 \\
\hline & CMP & -87.5 & 0.582 \\
\hline & $\mathrm{SH}$ & 414.5 & 0.020 \\
\hline & DBS-LF & 37.0 & 0.829 \\
\hline & \multicolumn{2}{|l|}{$\mathrm{NaCl}$} & \\
\hline \multirow[t]{4}{*}{ Non-visited quadrants in $5 \mathrm{~min}$} & $Q P$ & 6.8 & 0.001 \\
\hline & CMP & 2.3 & 0.216 \\
\hline & SH & -1.2 & 0.544 \\
\hline & DBS-LF & 2.1 & 0.296 \\
\hline \multirow[t]{5}{*}{ External/internal quadrants pathway } & $\mathrm{NaCl}$ & 1.96 & \multirow{5}{*}{$x$} \\
\hline & $Q P$ & 4.46 & \\
\hline & CMP & 3.10 & \\
\hline & $\mathrm{SH}$ & 1.19 & \\
\hline & DBS-LF & 1.44 & \\
\hline
\end{tabular}

Differences $(\Delta)$ between the means of distance traveled in centimeters of the $\mathrm{NaCl}$ group versus each of the other groups. There is a significant difference only between the groups $\mathrm{NaCl}$ versus $\mathrm{QP}(p=0.016)$ and $\mathrm{NaCl}$ versus $\mathrm{SH}(p=0.020)$. In the first case, the route decreased inversely to $390 \mathrm{~cm}$, in the second case, the same variable increased $>400 \mathrm{~cm}$. The presence of negative sign (-) means a decrease in the traveled distance between each group. There is no significant difference between the $\mathrm{NaCl}$ and CMP or DBS-LF groups. There is a difference in the number of quadrants not visited only significantly in the $\mathrm{NaCl}$ group when compared against $\mathrm{QP}$ ( $p=0.001$ ). When establishing a ratio between the visited external quadrants over the internal ones, it is observed that for the $\mathrm{NaCl}$ group, there are almost twice as many visits in the former over the latter (1.96) it explains the normal behavior of the rat at the OF which is moving along the edges, whereas for the QP group, this proportion is doubled which explain PB. The DBS-LF group (1.44) is very similar to the NaCl group recovering from normal behavior. A Leven test was used to determine homoscedasticity and an ANOVA test with post hoc DMS and Tamhane. Statistical analysis was done with IBM SPSS Statistics V21.0 software. CMP: clomipramine, DBS-LF: deep brain stimulation low frequency, NaCl: sodium chloride, QP: quinpirole, OF: open field, SH: sham (Electrode implant without electrostimulation), X: no comparison was made for these groups.

Homemade electrodes $(26 \mathrm{~mm}$ cannula and stainless steel cable coated with a $9 \mathrm{~mm}$ long insulating varnish, with the minimum resistance of $100 \mathrm{~K} \Omega$ ) were implanted. OFC coordinates were calculated from the Paxinos and Watson Atlas at $3.7 \mathrm{~mm}$ anterior to bregma, $2.4 \mathrm{~mm}$ lateral to the midline, and $4.8 \mathrm{~mm}$ ventral to the dura mater $^{21}$. After 3 days of recovery, DBS was performed with a Grass Instruments S88 Dual Output Pulse Stimulator (Grass Instruments Co, West Warwick, USA).

For this study, 40 rats were divided into the following groups: Group $\mathrm{NaCl}: n=9$, Group QP $n=12$, Group CMP $n=11$, Group SH, and Group DBS-LF $n=8$. The treatment and procedures applied to each group are shown in the following diagram.

\section{Results}

The distance traveled in $5 \mathrm{~min}$ in $\mathrm{cm}$ was used as the main output variable. In addition, the number of unvisited quadrants and the proportion of external quadrants to internal quadrants in the same period were reported
(Table 1). To know the distance traveled by each rat, it was determined as the movement of locomotion when the rat moved its entire body from one quadrant to another (the four legs cross the line that divides the quadrant). For the interpretation of this movement, video recordings of each maneuver were used, the locomotion routes were marked with a permanent marker on an acetate film placed on the computer screen. Once these marked routes were established, it was possible to count how many quadrants $(10 \mathrm{~cm} \times 10 \mathrm{~cm})$ the rat went through and interpreted as the $\mathrm{cm}$ traveled in $5 \mathrm{~min}$.

The $\mathrm{NaCl}$ group obtained an average of $656.6 \mathrm{~cm}, \mathrm{QP}$ group obtained an average of $266.6 \mathrm{~cm}$, and CMP group obtained an average of $569 \mathrm{~cm}$ this group corresponds to the conventional treatment OCD (gold standard), which decreases perseverative behavior in rats. $\mathrm{SH}$ group obtained an average of $1071.2 \mathrm{~cm}$, DBS-LF group (LF-left side) obtained an average of $693.8 \mathrm{~cm}$ and LFright side obtained an average of $842.5 \mathrm{~cm}$, altogether; both groups presented a greater amount of $\mathrm{cm}$ in comparison to the group $\mathrm{NaCl}$. In the statistical analysis, we 


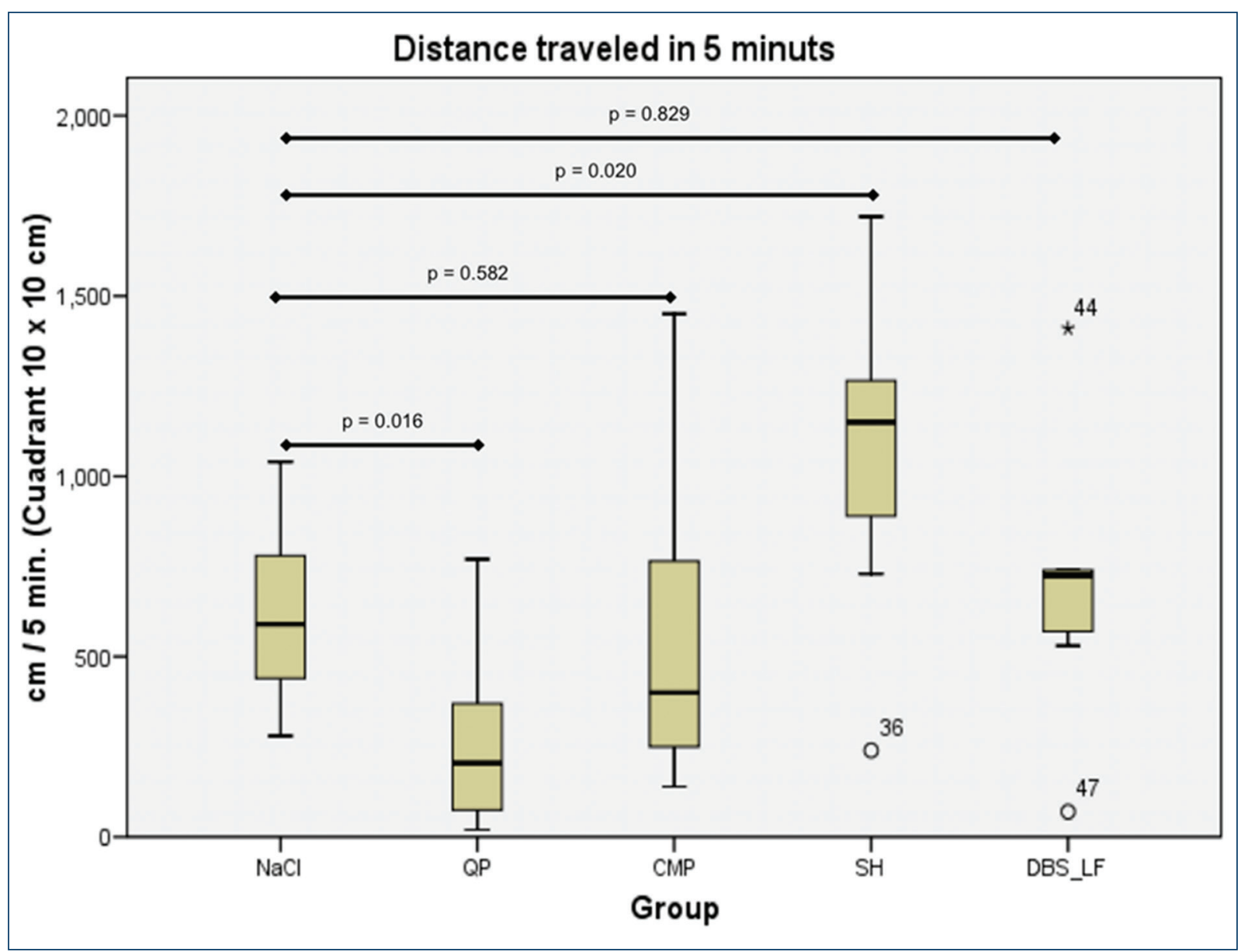

Figure 2. In this box plot, we can see the median $\mathrm{cm}$ traveled and their interquartile distributions and it is congruent with the $\Delta$ expressed in table 1. A decrease in motor behavior was observed for the QP group with a tendency to lead the median to low values that are interpreted as perseverative behavior, while the other groups are similar to the $\mathrm{NaCl}$ group, except the $\mathrm{SH}$ group that shows a hyperactive behavior.

can observe that when making multiple comparisons, it was found that there is no significant difference between DBS-LF-left side and DBS-LF-right side ( $p=1.00)$.

When comparing the distance traveled (Fig. 2) in the $\mathrm{NaCl}$ group with the groups treated with QP $(p=0.016)$ and SH group ( $p=0.020)$, a statistical difference was found, indicating the animal model was reproduced; however, when comparing $\mathrm{NaCl}$ group with CMP ( $p=0.582$ ), no significant differences were found, which was expected since it is the gold standard treatment. Finally, between $\mathrm{NaCl}$ and DBS-LF, no significant difference was found, the perseverative behavior decreased with this procedure.

\section{Discussion}

This study investigates whether DBS-LF decreases the perseverative behavior in the rat model of perseverative behavior induced by QP. The results revealed that
DBS-LF reduced perseverative behavior significantly in rats. The QP-induced checking behavior is a well-established rodent model that has many features of human compulsive checking. Santoyo et al. replicated the animal model with $8 \mathrm{OH}$ DPAT a 5 HT2 agonist that induces perseverative behavior in $\mathrm{T}$ maze apparatus and concluded that $90 \%$ of the rats decreased perseverative behavior with DBS-LF $(2 \mathrm{~Hz})$ in OFC ${ }^{22}$. Some authors ${ }^{23}$ use the number of visits to the home base rats as a variable, which consists of determining how many times the rodent visits a place in the open field, a site predetermined by the researcher, i.e., a housing cage. It can also be measured by how often rodents come into contact with objects placed at different points of the open field. The home base model can explain better the perseverative behavior naturally, but it also promotes memorization and reinforcement. In our study, although the rat is not in a natural environment, we eliminate home base and the visit objects to avoid memory bias 
or reinforcement. However, for the apparatus that we used the rats could at first seek an outlet to escape, with a tendency to take refuge in the edges, unlike the rats treated with $\mathrm{QP}, \mathrm{NaCl}$ group has a more uniform distribution of locomotion compared to QP group, which have a tendency to stay longer in certain places performing grooming, yawning, and licking.

The weaknesses of this study could be focused on not applying DBS-LF bilaterally at the same time and not having brain histology available to corroborate misplaced electrodes. Previous studies showed that bilateral low frequency can avoid perseverative behavior in an $8 \mathrm{OH}$-DPAT mode ${ }^{14,22}$. Seeman et al. concluded that the administration of ionotropic glutamate receptor antagonists such as phencyclidine, ketamine, and dizocilpine binds to dopamine $\mathrm{D} 2$ receptors, which may condition competitive agonist with QP at D2/D3 receptors during anesthetic procedure, thus modifying the behavior of the rat ${ }^{24}$.

Gök et al. reported that the administration of the metabotropic antagonist 5 receptors of glutamate named 2-methyl-6-phenylethynyl-pyridine (1 or 5 mg kg-1, i.p.) before each QP injection inhibited significantly the increase of locomotion and compulsive control behavior of rats ${ }^{25}$.

QP or a 5-HTA2 agonist such as 8-OH-DPAT can induce perseverative behavior, respectively, in open field and T-maze test by acting on different parts of the underlying motivation safety circuit in OCD. QP acts directly through dopaminergic pathways, motivating a checking activity, and 8-OH-DPAT perpetuates motivational activity by inhibition of negative feedback pathway.

Perseveration of routes and hyperactivity observed in the $\mathrm{SH}$ group could be the mechanic effect of the electrode associated to $\mathrm{QP}^{26}$. Comparing the $\mathrm{NaCl}$ and $\mathrm{SH}$ groups, we found that there was a significant difference in the distance traveled in 5 min between these groups $(p=0.020)$. In this study, the electrode implantation itself is capable to produce a change in the behavior of the rat; this change may be due to the microinjury effect $^{27-29}$ and QP pharmacokinetics (hyperkinetic phase). Hartesveldt proposes that QP has the capacity to induce suppressive effects on locomotion and the ability to cause an increase in motor activity ${ }^{30}$.

According to the results of this study, unilateral DBSLF can reduce the perseverative behavior in a QP model. On the other hand, Sturm et al. have published unilateral DBS in accumbens nucleus can reduce OCD symptoms in patients ${ }^{18}$. Specific clinical trials should be considered to get enough information on the safety and effectiveness of unilateral DBS in human being.

\section{Conclusions}

Chronic QP (0.5 mg/kg subcutaneous twice weekly for 5 weeks) produces perseverative behavior in Wistar rats; this behavior is manifested by a decrease in locomotion and an increase of the visits of certain quadrants of the open field.

Unilateral DBS-LF decreases perseverative behavior in Wistar rats induced by QP animal model in an open field test.

Dopamine plays a role in the development of perseverative behavior in the rat and can be modulated by electrostimulation at low frequency $(6 \mathrm{~Hz})$.

\section{Acknowledgments}

The authors would like to thank Ultralab for donating QP.

\section{Conflicts of interest}

The authors declare that they have no conflicts of interest.

\section{References}

1. Zike I, Xu T, Hong N, Veenstra-VanderWeele J. Rodent models of obsessive compulsive disorder: evaluating validity to interpret emerging neurobiology. Neuroscience. 2017;345:256-73.

2. Rojas CC, Jiménez-Ponce F, Flores-Vargas A, García AP. OCD in animal models using quinpirole as dopaminergic inductor of perseverative behaviour. Rev Méd Del Hosp Gen Méx. 2015;78:169-76.

3. Rodrigo B, Jorge A, Néstor M, et al. Quinpirole effects on the dopaminergic system. J Pharmacol Toxicol. 2011;2:310-7.

4. Novak G, Seeman P, Le Foll B. Exposure to nicotine produces an increase in dopamine D2(High) receptors: a possible mechanism for dopamine hypersensitivity. Int J Neurosci. 2010;120:691-7.

5. Dvorkin A, Silva C, McMurran T, et al. Features of compulsive checking behavior mediated by nucleus accumbens and orbital frontal cortex. Eur J Neurosci. 2010;32:1552-63.

6. Manning EE. There is much to be learned from animal models of obsessive-compulsive disorder. Biol Psychiatry. 2016;79:e1-3.

7. Ahmari SE, Spellman T, Douglass NL, et al. Repeated cortico-striatal stimulation generates persistent OCD-like behavior. Science. 2013;340:1234-9.

8. Burguière $E$, Monteiro $P$, Feng G, Graybiel AM. Optogenetic stimulation of lateral orbitofronto-striatal pathway suppresses compulsive behaviors. Science. 2013;340:1243-6.

9. de Haas R, Seddik A, Oppelaar H, Westenberg HG, Kas MJ. Marked inbred mouse strain difference in the expression of quinpirole induced compulsive like behavior based on behavioral pattern analysis. Eur Neuropsychopharmacol. 2012;22:657-63.

10. Schmidt TT, Rea E, Shababi-Klein J, Panagis G, Winter C. Enhanced reward-facilitating effects of $d$-amphetamine in rats in the quinpirole model of obsessive-compulsive disorder. Int $\mathrm{J}$ Neuropsychopharmacol. 2013;16:1083-91.

11. McCracken $C B$, Grace AA. High-frequency deep brain stimulation of the nucleus accumbens region suppresses neuronal activity and selectively modulates afferent drive in rat orbitofrontal cortex in vivo. J Neurosci. 2007;27:12601-10.

12. Klavir O, Flash S, Winter C, Joel D. High frequency stimulation and pharmacological inactivation of the subthalamic nucleus reduces 'compulsive' lever-pressing in rats. Exp Neurol. 2009;215:101-9.

13. Klavir O, Winter C, Daphna J. High but not lower frecuency stimulation of both the globus pallidus and the etopenduncular nucleus reduces "compulsive" lever-pressing in rats. Behav Brain Res. 2011;216:84-93.

14. Andrade $P$, Carrillo-Ruiz JD, Ramírez $Y$, Jiménez F. Effects of thalamic reticular nucleus electrical stimulation in rats in a T-maze perseverative behavior model induced by 8-OH-DPAT. Neuromodulation. 2010;13:2-9. 
15. Alonso $P$, Cuadras $D$, Gabriëls $L$, et al. Deep brain stimulation for obsessive-compulsive disorder: a meta-analysis of treatment outcome and predictors of response. PLoS One. 2015;10:e0133591.

16. Hirschtritt ME, Bloch MH, Mathews $\mathrm{CA}$. Obsessive-compulsive disorder: advances in diagnosis and treatment. JAMA. 2017;317:1358-67.

17. Fitzgerald PB, Segrave RA. Deep brain stimulation in mental health: review of evidence for clinical efficacy. Aust N Z J Psychiatry. 2015;49 979-93.

18. Sturm V, Lenartz D, Koulousakis A, et al. The nucleus accumbens: a target for deep brain stimulation in obsessive-compulsive-and anxiety-disorders. J Chem Neuroanat. 2003;26:293-9.

19. Jiménez $F$, Nicolini $H$, Lozano AM, et al. Electrical stimulation of the inferior thalamic peduncle in the treatment of major depression and obsessive compulsive disorders. World Neurosurg. 2013;80:S30.e17-25.

20. Tsutsui R, Shinomiya K, Sendo T, Kitamura Y, Kamei C. Effects of the $5-\mathrm{HT}(1 \mathrm{~A})$ receptor agonist tandospirone on ACTH-induced sleep disturbance in rats. Biol Pharm Bull. 2015;38:884-8.

21. Paxinos G, Watson C. The Rat Brain in Stereotaxic Coordinate. New York: Academic Press; 2005.

22. Santoyo SB. El Efecto de la Estimulación Eléctrica, en Corteza Orbitofrontal, en Ratas con Conducta Perseverante Inducida por 8 OH-DPAT Como un Modelo de Trastorno Obsesivo Compulsivo, MS Thesis; 2010.
23. Alkhatib AH, Dvorkin-Gheva A, Szechtman H. Quinpirole and 8-OH-DPAT induce compulsive checking behavior in male rats by acting on different functional parts of an OCD neurocircuit. Behav Pharmacol. 2013;24:65-73.

24. Seeman $\mathrm{P}$, Guan $\mathrm{HC}$, Hirbec $\mathrm{H}$. Dopamine D2High receptors stimulated by phencyclidines, lysergic acid diethylamide, salvinorin $A$, and modafinil. Synapse. 2009;63:698-704.

25. Gök S, M Demet ZM. Glutamate mGlu5 receptor antagonist, MPEP, reduces the quinpirole induced compulsive-like checking in rats. J Obsessive Compuls Relat Disord. 2017;15:13-8.

26. Eilam D, Golani I, Szechtman H. D2-agonist quinpirole induces perseveration of routes and hyperactivity but no perseveration of movements. Brain Res. 1989;490:255-67.

27. Jiménez $F$, Velasco F, Salin-Pascual $R$, et al. A patient with a resistant major depression disorder treated with deep brain stimulation in the inferior thalamic peduncle. Neurosurgery. 2005;57:585-93.

28. Pourfar M, Tang C, Lin T, et al. Assessing the microlesion effect of subthalamic deep brain stimulation surgery with FDG PET. J Neurosurg. 2009;110:1278-82

29. McCreery DB, Yuen TG, Agnew WF, Bullara LA. Stimulus parameters affecting tissue injury during microstimulation in the cochlear nucleus of the cat. Hear Res. 1994;77:105-15.

30. Van Hartesveldt C. Temporal and environmental effects on quinpirole-induced biphasic locomotion in rats. Pharmacol Biochem Behav. 1997;58:955-60. 